\title{
A Full Nesterov-Todd Step Feasible Weighted Primal-Dual Interior-Point Algorithm for Symmetric Optimization
}

\author{
Behrouz Kheirfam
}

Received: 15 September 2013 / Revised: 16 November 2013 / Accepted: 23 November 2013 /

Published online: 10 December 2013

(C) Operations Research Society of China, Periodicals Agency of Shanghai University, and

Springer-Verlag Berlin Heidelberg 2013

\begin{abstract}
In this paper a weighted short-step primal-dual interior-point algorithm for linear optimization over symmetric cones is proposed that uses new search directions. The algorithm uses at each interior-point iteration a full Nesterov-Todd step and the strategy of the central path to obtain a solution of symmetric optimization. We establish the iteration bound for the algorithm, which matches the currently best-known iteration bound for these methods, and prove that the algorithm is quadratically convergent.
\end{abstract}

Keywords Symmetric cone optimization · Full Nesterov-Todd step ·

Target-following $\cdot$ Polynomial complexity

Mathematics Subject Classification (2000) 90C51 · 90C33

\section{Introduction}

Let $(\mathcal{J}, \circ)$ be an Euclidean Jordan Algebra (EJA) with rank $r$ equipped with the standard inner product $\langle x, y\rangle=\operatorname{Tr}(x \circ y)$. Let $\mathcal{K}$ be the corresponding symmetric cone. We consider the symmetric cone optimization (SCO) problem given in the standard form

$$
\min \{\langle c, x\rangle: A x=b, x \in \mathcal{K}\},
$$

and its dual problem

$$
\max \left\{b^{T} y: A^{T} y+s=c, s \in \mathcal{K}\right\},
$$

where $c$ and the rows of $A$ lie in $\mathcal{J}$, and $b \in \mathbb{R}^{m}$. We assume that the matrix $A$ has full rank, i.e., $\operatorname{rank}(A)=m$.

B. Kheirfam $(\bowtie)$

Department of Mathematics, Azerbaijan Shahid Madani University, Tabriz, Iran

e-mail: b.kheirfam@azaruniv.edu 
In recent years it has been established that symmetric cones which are cones of squares of EJAs, serve as a unifying framework to which the important cases of cones used in optimization. The classical monograph of Faraut and Korányi [6] provides a wealth of information on Jordan algebras, symmetric cones and related topics.

Nesterov and Todd $[14,15]$ provided a theoretical foundation for efficient primaldual interior-point methods (IPMs) on a special class of convex optimization problems, where the associated cone was self-scaled. However, they did not make the connection to EJAs. The first work connecting EJAs and optimization is due to Güler [10]. He observed that the family of the self-scaled cones is identical to the set of symmetric cones for which there exists a complete classification theory. The application of the EJA as a basic tool for analyzing complexity proofs of the IPMs for SCO and SCLCP was started by Faybusovich [8], who extended earlier works of Nesterov and Todd, and Kojima et al. [13, 15]. Schmieta and Alizadeh [16, 17] studied primal-dual IPMs for SCO extensively under the framework of EJA. Rangarajan [19] proved the polynomial-time convergence of infeasible IPMs for conic programming over symmetric cones using a wide neighborhood of the central path for a commutative family of search directions. Vieira [21] proposed primal-dual IPMs for SCO based on the so-called eligible kernel functions and obtained the currently best-known iteration bounds for the large- and small-update methods. Darvay [3] proposed a full-Newton step primal-dual interior-point algorithm for LO. The search direction of his algorithm is introduced by using an algebraic equivalent transformation of the nonlinear equations which define the central path and then applying Newton's method for the new system of equations. Later on, Wang and Bai [23-25], Wang [22] and Wang et al. [27], respectively, extended Darvay's algorithm for LO to second-order cone optimization (SOCO), semi-definite optimization (SDO), symmetric cone optimization (SCO), monotone linear complementarity problem over symmetric cones and convex quadratic optimization over symmetric cone. Very recent, Kheirfam [12] proposed a full Nesterov-Todd step infeasible IPM (IIPM) based on Darvay's technique for $\mathrm{SCO}$, and proved that the complexity of the algorithm coincides with the best-known iteration bound for IIPMs.

Feasible primal-dual path-following methods are an important class of IPMs due to their numerical efficiency and theoretical polynomial complexity. These methods used the so-called central path as a guideline to obtain a solution of SCO. However, the theoretical issues of these methods show that they require that starting primaldual points must be strictly feasible and closed to the central path. Still, it is uneasy to find suitable centered strictly feasible starting points. Therefore, it is worth analyzing other cases when points are not on the central path. Target-following primal-dual IPMs belong to the strategies to overcome this restriction. These methods are based on the observation that with every central-path algorithm we can associate a target sequence on the central path. For a study of comprehension we refer the reader to [11]. Weighted-path-following methods can be viewed as a particular case of targetfollowing methods $[1,4,11]$. In contrast with classical path-following methods, one multi-dimensional parameter $\bar{v}$ is used instead of the one dimensional parameter $\mu$. These methods were studied by Ding and Li [5] for monotone linear complementarity problem (LCP), and by Roos and Hertog [20] for linear optimization (LO). Later on, Darvay [4], Achache [1] and Wang et al. [26], respectively, extended it for LO, mono- 
tone LCP and monotone horizontal LCP by using new search directions of Newton method.

The purpose of the paper is to generalize Darvay's weighted-path-following algorithm for LO to SCO by using EJA. At each iteration, we use only full Nesterov-Todd steps (NT) which have the advantage that no line searches are needed. We derive the complexity bound.

The paper is organized as follows: In the next section, we briefly introduce the theory of the Euclidean Jordan algebras and their associated symmetric cones, which are needed in this paper. In Sect. 3, after briefly reviewing the concept of the central path for SCO, we provide the target-following search directions for SCO. In Sect. 4, we present the weighted-path-following algorithm for SCO. In Sect. 5, we analyze the algorithm and derive the complexity bound. In Sect. 6, a numerical result is reported. Finally, some conclusions follow in Sect. 7.

\section{Euclidean Jordan Algebra}

A Jordan algebra $\mathcal{J}$ is a finite dimensional vector space endowed with a bilinear map $\circ: \mathcal{J} \times \mathcal{J} \rightarrow \mathcal{J}$ if for all $x, y \in \mathcal{J}, x \circ y=y \circ x$, and $x \circ\left(x^{2} \circ y\right)=x^{2} \circ(x \circ y)$, where $x^{2}=x \circ x$. A Jordan algebra $(\mathcal{J}, \circ)$ is called Euclidean if there exists a symmetric positive definite quadratic form $P$ on $\mathcal{J}$ such that $P(x \circ y, z)=P(x, y \circ z)$. A Jordan algebra has an identity element, if there exists a unique element $e \in \mathcal{J}$ such that $x \circ e=e \circ x=x$, for all $x \in \mathcal{J}$. The set $\mathcal{K}:=\mathcal{K}(\mathcal{J})=\left\{x^{2}: x \in \mathcal{J}\right\}$ is called the cone of squares of Euclidean Jordan algebra $(\mathcal{J}, \circ,\langle.,\rangle$.$) . A cone is symmetric if and only$ if it is the cone of squares of an EJA [6].

An element $c \in \mathcal{J}$ is idempotent if $c \circ c=c$. An idempotent $c$ is primitive if it is nonzero and can not be expressed by the sum of two other nonzero idempotents. A set of primitive idempotents $\left\{c_{1}, c_{2}, \cdots, c_{k}\right\}$ is called a Jordan frame if $c_{i} \circ c_{j}=0$, for any $i \neq j \in\{1,2, \cdots, k\}$ and $\sum_{i=1}^{k} c_{i}=e$. For any $x \in \mathcal{J}$, let $r$ be the smallest positive integer such that $\left\{e, x, x^{2}, \cdots, x^{r}\right\}$ is linearly dependent; $r$ is called the degree of $x$ and is denoted by $\operatorname{deg}(x)$. The rank of $\mathcal{J}$, denoted by $\operatorname{rank}(\mathcal{J})$, is defined as the maximum of $\operatorname{deg}(x)$ over all $x \in \mathcal{J}$.

Theorem 2.1 (Theorem III.1.2 in [6]) Let $(\mathcal{J}, \circ,\langle.,\rangle$.$) be a Euclidean Jordan$ algebra with rank $(\mathcal{J})=r$. Then, for any $x \in \mathcal{J}$, there exists a Jordan frame $\left\{c_{1}, c_{2}, \cdots, c_{r}\right\}$ and real numbers $\lambda_{1}(x), \lambda_{2}(x), \cdots, \lambda_{r}(x)$ such that $x=$ $\sum_{i=1}^{r} \lambda_{i}(x) c_{i}$.

Every $\lambda_{i}(x)$ is called an eigenvalue of $x$. We denote $\lambda_{\min }(x)\left(\lambda_{\max }(x)\right)$ as the minimal (maximal) eigenvalue of $x$. We can define the following: the square root, $x^{\frac{1}{2}}:=\sum_{i=1}^{r} \sqrt{\lambda_{i}(x)} c_{i}$, wherever all $\lambda_{i} \geqslant 0$, the inverse, $x^{-1}:=\sum_{i=1}^{r} \lambda_{i}(x)^{-1} c_{i}$, wherever all $\lambda_{i} \neq 0$, the square $x^{2}:=\sum_{i=1}^{r} \lambda_{i}(x)^{2} c_{i}$, the trace $\operatorname{Tr}(x):=\sum_{i=1}^{r} \lambda_{i}(x)$.

Since "o" is bilinear map, for every $x \in \mathcal{J}$, a linear operator $L(x)$ can be defined such that $L(x) y=x \circ y$ for all $y \in \mathcal{J}$. In particular, $L(x) e=x$ and $L(x) x=x^{2}$. For each $x \in \mathcal{J}$, define

$$
P(x):=2 L(x)^{2}-L\left(x^{2}\right),
$$


where $L(x)^{2}=L(x) L(x)$. The map $P(x)$ is called the quadratic representation of $x$. For any $x, y \in \mathcal{J}, x$ and $y$ are said to be operator commutable if $L(x)$ and $L(y)$ commute, i.e., $L(x) L(y)=L(y) L(x)$. In other words, $x$ and $y$ operator commutable if for all $z \in \mathcal{J}, x \circ(y \circ z)=y \circ(x \circ z)$ (see [16]). For any $x, y \in \mathcal{J}$, the inner product is defined as $\langle x, y\rangle=\operatorname{Tr}(x \circ y)$, and the Frobenius norm of $x$ as follows: $\|x\|_{F}=\sqrt{\operatorname{Tr}\left(x^{2}\right)}=\sqrt{\sum_{i=1}^{r} \lambda_{i}^{2}(x)}$. Observe that $\|e\|_{F}=\sqrt{r}$, since identity element $e$ has eigenvalue 1 .

Lemma 2.1 (Lemma 3.2 in [7]) Let $x, s \in$ int $\mathcal{K}$. Then, there exists a unique $w \in$ int $\mathcal{K}$ such that

$$
x=P(w) s .
$$

Moreover,

$$
w=P\left(x^{\frac{1}{2}}\right)\left(P\left(x^{\frac{1}{2}}\right) s\right)^{-\frac{1}{2}}=P\left(s^{-\frac{1}{2}}\right)\left(P\left(s^{\frac{1}{2}}\right) x\right)^{\frac{1}{2}} .
$$

The point $w$ is called the scaling point of $x$ and $s$. Hence, there exists $\tilde{v} \in$ int $\mathcal{K}$ such that

$$
\tilde{v}=P(w)^{-\frac{1}{2}} x=P(w)^{\frac{1}{2}} s,
$$

which is the so-called NT-scaling of $\mathbb{R}^{n}$.

Let $x, y \in \mathcal{J}$. We say that two elements $x$ and $y$ are similar, as denoted by $x \sim y$, if and only if $x$ and $y$ share the same set of eigenvalues. We say $x \in \mathcal{K}$ if and only if $\lambda_{i} \geqslant 0$ and $x \in \operatorname{int} \mathcal{K}$ if and only if $\lambda_{i}>0$, for all $i=1,2, \cdots, r$. We also say $x$ is positive semi-definite (positive definite) if $x \in \mathcal{K}(x \in$ int $\mathcal{K})$.

Here, we list some results which needed in this paper.

Lemma 2.2 (Proposition 2.1 in [16]) Let $x, s, u \in$ int $\mathcal{K}$. Then

(i) $P\left(x^{\frac{1}{2}}\right) s \sim P\left(s^{\frac{1}{2}}\right) x$.

(ii) $P\left((P(u) x)^{\frac{1}{2}}\right) P\left(u^{-1}\right) s \sim P\left(x^{\frac{1}{2}}\right) s$.

Lemma 2.3 (Proposition 3.2.4 in [21]) Let $x, s \in$ int $\mathcal{K}$, and $w$ be the scaling point of $x$ and $s$. Then

$$
\left(P\left(x^{\frac{1}{2}}\right) s\right)^{\frac{1}{2}} \sim P\left(w^{\frac{1}{2}}\right) s .
$$

Lemma 2.4 (Theorem 4 in [18]) Let $x, s \in$ int $\mathcal{K}$. Then

$$
\lambda_{\min }\left(P(x)^{\frac{1}{2}} s\right) \geqslant \lambda_{\min }(x \circ s) .
$$

Lemma 2.5 (Lemma 4.1 in [25]) Let $x(\alpha)=x+\alpha \Delta x, s(\alpha)=s+\alpha \Delta s$ for $0 \leqslant \alpha \leqslant$ 1 with $x, s \in$ int $\mathcal{K}$ and $x(\alpha) \circ s(\alpha) \in \operatorname{int} \mathcal{K}$ for $\alpha \in[0, \bar{\alpha}]$. Then, $x(\bar{\alpha}) \in$ int $\mathcal{K}$ and $s(\bar{\alpha}) \in \operatorname{int} \mathcal{K}$. 


\section{The Central Path and New Search Directions}

Throughout the paper, we assume that both (CP) and (CD) satisfy the interior-point condition (IPC), i.e., there exist $x^{0} \in \operatorname{int} \mathcal{K}$ and $s^{0} \in \operatorname{int} \mathcal{K}$ such that $A x^{0}=b$ and $A^{T} y^{0}+s^{0}=c$. Under the IPC, finding an optimal solution of (CP) and (CD) is equivalent to solving the following system:

$$
\begin{aligned}
A x & =b, \quad x \in \mathcal{K}, \\
A^{T} y+s & =c, \quad s \in \mathcal{K}, \\
x \circ s & =0 .
\end{aligned}
$$

The basic idea of primal-dual IPMs is to replace the third equation in (3.1), the socalled complementarity condition for (CP) and (CD), by the parameterized equation $x \circ s=\mu e$, with $\mu>0$. Thus, one may consider

$$
\begin{aligned}
A x & =b, \quad x \in \mathcal{K}, \\
A^{T} y+s & =c, \quad s \in \mathcal{K}, \\
x \circ s & =\mu e .
\end{aligned}
$$

For each $\mu>0$, the system (3.2) has a unique solution $(x(\mu), y(\mu), s(\mu))$, and we call $x(\mu)$ and $(y(\mu), s(\mu))$ the $\mu$-center of (CP) and (CD), respectively. The set of $\mu$-centers (with $\mu$ running through all positive real numbers) gives a homotopy path, which is called the central path of (CP) and (CD) [9]. The target-following approach starts from the observation that the system (3.2) can be generalized by replacing the vector $\mu e$ with an arbitrary positive vector $\bar{v}^{2}$. Thus we obtain the following system:

$$
\begin{aligned}
A x & =b, \quad x \in \mathcal{K}, \\
A^{T} y+s & =c, \quad s \in \mathcal{K}, \\
x \circ s & =\bar{v}^{2},
\end{aligned}
$$

where $\bar{v}>0$. It is known that there exists one unique solution denoted by $(x(\bar{v}), y(\bar{v})$, $s(\bar{v}))$ of (3.3) [2]. The path $\bar{v} \rightarrow(x(\bar{v}), y(\bar{v}), s(\bar{v}))$ is called the weighted path of (CP) and (CD). If $\bar{v}$ goes to zero, then the limit of the weighted path exists and since the limit point satisfies the complementarity condition, the limit yields a solution for (CP) and $(\mathrm{CD})$.

Remark 3.1 If $\bar{v}^{2}=\mu e$ with $\mu>0$, then the weighted path coincides with the classical central path.

Now we propose a new search direction for (CP) and (CD) based on Darvay's method for LO [4]. Consider the function

$$
\varphi \in C^{1}, \quad \varphi: \mathbb{R}^{+} \rightarrow \mathbb{R}^{+},
$$


and suppose that the inverse function $\varphi^{-1}$ exists. Then, the system (3.3) can be written in the following equivalent form:

$$
\begin{aligned}
A x & =b, \quad x \in \mathcal{K}, \\
A^{T} y+s & =c, \quad s \in \mathcal{K}, \\
\varphi(x \circ s) & =\varphi\left(\bar{v}^{2}\right) .
\end{aligned}
$$

We apply Newton's method to this system, and obtain the following relations for the corresponding displacements in the $x-, y$ - and $s$-spaces:

$$
\begin{aligned}
A \Delta x & =0, \\
A^{T} \Delta y+\Delta s & =0, \\
x \circ \Delta s+s \circ \Delta x & =\left(\varphi^{\prime}(x \circ s)\right)^{-1} \circ\left(\varphi\left(\bar{v}^{2}\right)-\varphi(x \circ s)\right) .
\end{aligned}
$$

Due to the fact that $x$ and $s$ do not operator commute, in general, i.e., $L(x) L(s) \neq$ $L(s) L(x)$, the system (3.5) does not always have a unique solution. It is now well known that this difficulty can be resolved by applying a scaling scheme. This is given in the following lemma.

Lemma 3.1 (Lemma 28 in [16]) Let $u \in \operatorname{int} \mathcal{K}$. Then

$$
x \circ s=\mu e \quad \Leftrightarrow \quad P(u) x \circ P(u)^{-1} s=\mu e .
$$

Now, replacing the third equation in (3.4) by $\varphi\left(P(u) x \circ P\left(u^{-1}\right) s\right)=\varphi\left(\bar{v}^{2}\right)$, and then applying Newton's method, we obtain the system

$$
\begin{aligned}
& A \Delta x=0, \\
& A^{T} \Delta y+\Delta s=0 \\
& P(u) x \circ P(u)^{-1} \Delta s+P(u)^{-1} s \circ P(u) \Delta x \\
& \quad=\left(\varphi^{\prime}\left(P(u) x \circ P(u)^{-1} s\right)\right)^{-1} \circ\left(\varphi\left(\bar{v}^{2}\right)-\varphi\left(P(u) x \circ P(u)^{-1} s\right)\right) .
\end{aligned}
$$

Here, we consider the NT-scaling scheme [15]. Let $u=w^{-\frac{1}{2}}$, where $w$ is the NTscaling point of $x$ and $s$ as defined in Lemma 2.1. We define

$$
v:=P(w)^{\frac{1}{2}} s\left[=P(w)^{-\frac{1}{2}} x\right]
$$

and

$$
\bar{A}:=A P(w)^{\frac{1}{2}}, \quad d_{x}:=P(w)^{-\frac{1}{2}} \Delta x, \quad d_{s}:=P(w)^{\frac{1}{2}} \Delta s .
$$

Using (3.7) and (3.8), after some elementary reductions, we obtain

$$
\bar{A} d_{x}=0,
$$




$$
\begin{aligned}
\bar{A}^{T} \Delta y+d_{s} & =0, \\
d_{x}+d_{s} & =p_{v},
\end{aligned}
$$

where

$$
p_{v}=v^{-1} \circ \varphi^{\prime}\left(v^{2}\right)^{-1} \circ\left(\varphi\left(\bar{v}^{2}\right)-\varphi\left(v^{2}\right)\right) .
$$

It easily follows that the above system has a unique solution in int $\mathcal{K}$ [21]. Hence, this system uniquely defines the scaled directions $d_{x}$ and $d_{s}$. To get the search directions $\Delta x$ and $\Delta s$ in the original space, we simply transform the scaled search directions back to the $x$-space and $s$-space by using (3.8):

$$
\Delta x=P(w)^{\frac{1}{2}} d_{x}, \quad \Delta s=P(w)^{-\frac{1}{2}} d_{s} .
$$

The new iterate is obtained by taking a full NT-step as follows:

$$
x^{+}:=x+\Delta x, \quad y^{+}:=y+\Delta y, \quad s^{+}:=s+\Delta s .
$$

\section{An Interior-Point Algorithm}

In this section, we take $\varphi(t)=\sqrt{t}$ based on Darvay's technique for LO [4] and we develop a new weighted-path-following algorithm based on the appropriate search directions. In the analysis of target-following algorithm we will need a measure for the proximity of the current iterate $v$ to the current target point $\bar{v}$. For this purpose we introduce the following proximity measure:

$$
\sigma(v, \bar{v})=\frac{\left\|p_{v}\right\|_{F}}{2 \lambda_{\min }(\bar{v})}=\frac{\|\bar{v}-v\|_{F}}{\lambda_{\min }(\bar{v})},
$$

where

$$
p_{v}=2(\bar{v}-v), \quad \lambda_{\min }(\bar{v})=\min \left\{\lambda_{i}(\bar{v}): 1 \leqslant i \leqslant r\right\} .
$$

We also use the vector $q_{v}$, defined by

$$
q_{v}=d_{x}-d_{s} .
$$

Note that the orthogonality of $d_{x}$ and $d_{s},\left\langle d_{x}, d_{s}\right\rangle=0$, implies that

$$
\left\|p_{v}\right\|_{F}=\left\|q_{v}\right\|_{F}
$$

We also have

$$
d_{x}=\frac{p_{v}+q_{v}}{2}, \quad d_{s}=\frac{p_{v}-q_{v}}{2}, \quad d_{x} \circ d_{s}=\frac{p_{v} \circ p_{v}-q_{v} \circ q_{v}}{4} .
$$

The algorithm starts with $\left(v^{0}, \bar{v}^{0}\right)$ such that $\sigma\left(v^{0}, \bar{v}^{0}\right) \leqslant \tau$. In each iteration the search directions at the current iterates with respect to the current value of $\bar{v}$ be computed and then the new iterate is obtained. The algorithm terminates with a point that satisfies $\operatorname{Tr}(x \circ s) \leqslant \epsilon$. We summarize the steps of the algorithm as Algorithm 1 below. 


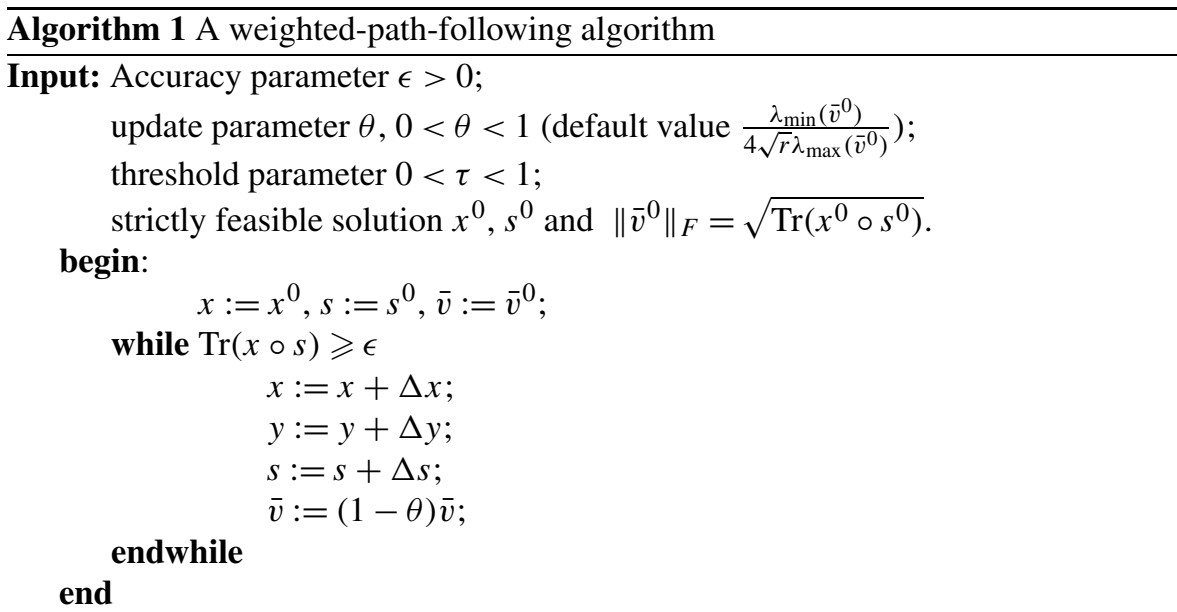

\section{Analysis of the Algorithm}

\subsection{Feasibility}

We proceed by investigating when the full NT-step to the target point $\bar{v}$ can be made without becoming infeasible. So, we want to know under which conditions the new iterates $x:=x+\Delta x$ and $s:=s+\Delta s$ are strictly positive. The next lemma gives a simple condition on $\sigma(v, \bar{v})$, which guarantees that the property is met after a full NT-step. For this purpose, from (3.7), (3.8) and (3.11) we have

$$
x^{+}=x+\Delta x=P(w)^{\frac{1}{2}}\left(v+d_{x}\right), \quad s^{+}=s+\Delta s=P(w)^{-\frac{1}{2}}\left(v+d_{s}\right) .
$$

Since $P(w)^{\frac{1}{2}}$ and $P(w)^{-\frac{1}{2}}$ are automorphisms of int $\mathcal{K}$ (Theorem III.2.1 in [6]), $x^{+}$ and $s^{+}$belong to int $\mathcal{K}$ if and only if $v+d_{x}$ and $v+d_{s}$ belong to int $\mathcal{K}$.

Lemma 5.1 The full NT-step is strictly feasible if $\sigma:=\sigma(v, \bar{v})<1$.

Proof Introduce a step length $\alpha$ with $\alpha \in[0,1]$ and define $v_{x}(\alpha)=v+\alpha d_{x}, v_{s}(\alpha)=$ $v+\alpha d_{s}$. Using the third equation of (3.9) and (4.3) we have

$$
\begin{aligned}
v_{x}(\alpha) \circ v_{s}(\alpha) & =v \circ v+\alpha v \circ\left(d_{x}+d_{s}\right)+\alpha^{2} d_{x} \circ d_{s} \\
& =v \circ v+\alpha v \circ p_{v}+\alpha^{2} \frac{p_{v} \circ p_{v}-q_{v} \circ q_{v}}{4} \\
& =(1-\alpha) v \circ v+\alpha\left(v \circ v+v \circ p_{v}\right)+\alpha^{2} \frac{p_{v} \circ p_{v}-q_{v} \circ q_{v}}{4} \\
& =(1-\alpha) v \circ v+\alpha\left(\bar{v} \circ \bar{v}-(1-\alpha) \frac{p_{v} \circ p_{v}}{4}-\alpha \frac{q_{v} \circ q_{v}}{4}\right),
\end{aligned}
$$

which the last equality holds by

$v \circ v+v \circ p_{v}=v \circ v+2 v \circ(\bar{v}-v)=\bar{v} \circ \bar{v}-(\bar{v}-v) \circ(\bar{v}-v)=\bar{v} \circ \bar{v}-\frac{p_{v} \circ p_{v}}{4}$. 
Furthermore, for $0 \leqslant \alpha \leqslant 1$, by the definition of $\sigma(v, \bar{v})$ and (5.1) we have

$$
\begin{aligned}
\left\|(1-\alpha) \frac{p_{v} \circ p_{v}}{4}+\alpha \frac{q_{v} \circ q_{v}}{4}\right\|_{F} & \leqslant(1-\alpha) \frac{\left\|p_{v} \circ p_{v}\right\|_{F}}{4}+\alpha \frac{\left\|q_{v} \circ q_{v}\right\|_{F}}{4} \\
& \leqslant(1-\alpha) \frac{\left\|p_{v}\right\|_{F}^{2}}{4}+\alpha \frac{\left\|q_{v}\right\|_{F}^{2}}{4} \\
& =(1-\alpha) \frac{\left\|p_{v}\right\|_{F}^{2}}{4}+\alpha \frac{\left\|p_{v}\right\|_{F}^{2}}{4} \\
& =\sigma^{2} \lambda_{\min }(\bar{v})^{2}<\lambda_{\min }(\bar{v})^{2},
\end{aligned}
$$

which shows that

$$
\bar{v} \circ \bar{v}-(1-\alpha) \frac{p_{v} \circ p_{v}}{4}-\alpha \frac{q_{v} \circ q_{v}}{4} \in \operatorname{int} \mathcal{K} .
$$

Thus

$$
(1-\alpha) v \circ v+\alpha\left(\bar{v} \circ \bar{v}-(1-\alpha) \frac{p_{v} \circ p_{v}}{4}-\alpha \frac{q_{v} \circ q_{v}}{4}\right) \in \operatorname{int} \mathcal{K} .
$$

If $0 \leqslant \alpha \leqslant 1$, then we have $v_{x}(\alpha) \circ v_{s}(\alpha) \in$ int $\mathcal{K}$. Hence, since $x, s \in$ int $\mathcal{K}$, Lemma 2.5 implies that $v_{x}(1)=v+d_{x} \in$ int $\mathcal{K}$ and $v_{s}(1)=v+d_{s} \in$ int $\mathcal{K}$. This completes the proof.

\subsection{Effect on the Proximity Measure}

According to (3.7), the $v$-vector after the step is given by

$$
v^{+}=P\left(w^{+}\right)^{-\frac{1}{2}} x^{+}\left[=P\left(w^{+}\right)^{\frac{1}{2}} s^{+}\right],
$$

where $w^{+}$is the scaling point of $x^{+}$and $s^{+}$. Using (5.1), Lemma 2.3 and Lemma 2.2, we have

$$
\begin{aligned}
\left(v^{+}\right)^{2} & \sim P\left(x^{+}\right)^{\frac{1}{2}} s^{+} \\
& =P\left(P(w)^{\frac{1}{2}}\left(v+d_{x}\right)\right)^{\frac{1}{2}} P(w)^{-\frac{1}{2}}\left(v+d_{s}\right) \\
& \sim P\left(v+d_{x}\right)^{\frac{1}{2}}\left(v+d_{s}\right) .
\end{aligned}
$$

Lemma 5.2 The following holds:

$$
\lambda_{\min }\left(v^{+}\right) \geqslant \lambda_{\min }(\bar{v}) \sqrt{1-\sigma^{2}} .
$$

Proof By using (5.3), Lemma 2.4, (4.3) and (5.1) with $\alpha=1$, we get

$$
\begin{aligned}
\lambda_{\min }\left(\left(v^{+}\right)^{2}\right) & =\lambda_{\min }\left(P\left(v+d_{x}\right)^{\frac{1}{2}}\left(v+d_{s}\right)\right) \\
& \geqslant \lambda_{\min }\left(\left(v+d_{x}\right) \circ\left(v+d_{s}\right)\right)
\end{aligned}
$$




$$
\begin{aligned}
& =\lambda_{\min }\left(\bar{v} \circ \bar{v}-\frac{q_{v} \circ q_{v}}{4}\right) \\
& \geqslant \lambda_{\min }(\bar{v})^{2}-\frac{\left\|q_{v}\right\|_{F}^{2}}{4} \\
& =\lambda_{\min }(\bar{v})^{2}-\frac{\left\|p_{v}\right\|_{F}^{2}}{4} \\
& =\lambda_{\min }(\bar{v})^{2}\left(1-\sigma^{2}\right) .
\end{aligned}
$$

This completes the proof.

We are interested in showing that closing a full NT-step brings us to the target point $\bar{v}$. In this respect the following lemma is of interest. It shows that if the current iterate $v$ is close enough to the target point $\bar{v}$, the full NT-step ensures quadratic convergence of the proximity measure.

Lemma 5.3 Let $\sigma:=\sigma(v, \bar{v})<1$. Then

$$
\sigma\left(v^{+}, \bar{v}\right) \leqslant \frac{\sigma^{2}}{1+\sqrt{1-\sigma^{2}}} .
$$

Thus $\sigma\left(v^{+}, \bar{v}\right) \leqslant \sigma^{2}$, which shows the quadratical convergence of the algorithm.

Proof From (5.1) with $\alpha=1$, we have

$$
\left(v+d_{x}\right) \circ\left(v+d_{s}\right)=\bar{v} \circ \bar{v}-\frac{q_{v} \circ q_{v}}{4} .
$$

Using (5.4), Lemma 5.2 and (4.3), we get

$$
\begin{aligned}
\sigma\left(v^{+}, \bar{v}\right) & =\frac{\left\|\bar{v}-v^{+}\right\|_{F}}{\lambda_{\min }(\bar{v})}=\frac{1}{\lambda_{\min }(\bar{v})}\left\|\frac{\bar{v} \circ \bar{v}-v^{+} \circ v^{+}}{\bar{v}+v^{+}}\right\|_{F} \\
& \leqslant \frac{\left\|\bar{v} \circ \bar{v}-\left(v+d_{x}\right) \circ\left(v+d_{S}\right)\right\|_{F}}{\lambda_{\min }(\bar{v})\left(\lambda_{\min }(\bar{v})+\lambda_{\min }\left(v^{+}\right)\right)} \\
& \leqslant \frac{\left\|q_{v} \circ q_{v}\right\|_{F}}{\left(2 \lambda_{\min }(\bar{v})\right)^{2}\left(1+\sqrt{1-\sigma^{2}}\right)} \\
& \leqslant \frac{\left\|q_{v}\right\|_{F}^{2}}{\left(2 \lambda_{\min }(\bar{v})\right)^{2}\left(1+\sqrt{1-\sigma^{2}}\right)} \\
& =\frac{\left\|p_{v}\right\|_{F}^{2}}{\left(2 \lambda_{\min }(\bar{v})\right)^{2}\left(1+\sqrt{1-\sigma^{2}}\right)} \\
& =\frac{\sigma^{2}}{1+\sqrt{1-\sigma^{2}}} .
\end{aligned}
$$

This completes the proof. 


\subsection{Effect on the Duality Gap}

The next lemma gives the effect of full NT-step on duality gap.

Lemma 5.4 Let the primal-dual feasible pair $\left(x^{+}, s^{+}\right)$be obtained after a full NTstep with respect to $\bar{v}$. Then, one has

$$
\left\langle x^{+}, s^{+}\right\rangle \leqslant\|\bar{v}\|_{F}^{2} .
$$

Proof Using (5.4), we have

$$
\begin{aligned}
\left\langle x^{+}, s^{+}\right\rangle=\left\langle v+d_{x}, v+d_{s}\right\rangle & =\operatorname{Tr}\left(\left(v+d_{x}\right) \circ\left(v+d_{s}\right)\right) \\
& =\operatorname{Tr}\left(\bar{v} \circ \bar{v}-\frac{q_{v} \circ q_{v}}{4}\right) \\
& =\|\bar{v}\|_{F}^{2}-\frac{1}{4}\left\|q_{v}\right\|_{F}^{2} \\
& \leqslant\|\bar{v}\|_{F}^{2} .
\end{aligned}
$$

The proof is complete.

\subsection{Updating the Target}

The following lemma describes the effect on the proximity measure of a full NT-step followed by an update in the target.

Theorem 5.1 Let $v$ and $\bar{v}$ be such that $\sigma:=\sigma(v, \bar{v})<1$ and $\bar{v}^{+}=(1-\theta) \bar{v}$, where $0<\theta<1$. Then

$$
\sigma\left(v^{+}, \bar{v}^{+}\right) \leqslant \frac{\theta \sqrt{r}}{1-\theta} \frac{\lambda_{\max }(\bar{v})}{\lambda_{\min }(\bar{v})}+\frac{\sigma^{2}}{(1-\theta)\left(1+\sqrt{1-\sigma^{2}}\right)} .
$$

Proof We have

$$
\begin{aligned}
\sigma\left(v^{+}, \bar{v}^{+}\right) & =\frac{1}{\lambda_{\min }\left(\bar{v}^{+}\right)}\left\|\bar{v}^{+}-v^{+}\right\|_{F} \\
& \leqslant \frac{1}{\lambda_{\min }\left(\bar{v}^{+}\right)}\left(\left\|\bar{v}^{+}-\bar{v}\right\|_{F}+\left\|\bar{v}-v^{+}\right\|_{F}\right) \\
& \leqslant \frac{\theta}{1-\theta} \frac{\|\bar{v}\|_{F}}{\lambda_{\min }(\bar{v})}+\frac{1}{1-\theta} \sigma\left(v^{+}, \bar{v}\right) \\
& \leqslant \frac{\theta \sqrt{r}}{1-\theta} \frac{\lambda_{\max }(\bar{v})}{\lambda_{\min }(\bar{v})}+\frac{\sigma^{2}}{(1-\theta)\left(1+\sqrt{1-\sigma^{2}}\right)} .
\end{aligned}
$$

This completes the proof. 


\subsection{The Choice of $\tau$ and $\theta$}

We intend to determine values of a threshold $\tau$ and an update parameter $\theta$, so that at the start of the iteration, we have $\sigma(v, \bar{v}) \leqslant \tau$. After the full NT-step and a step along the weighted path, the property $\sigma\left(v^{+}, \bar{v}^{+}\right) \leqslant \tau$ should be maintained. In this case, by Theorem 5.1, it suffices to have

$$
\frac{\theta \sqrt{r}}{1-\theta} \frac{\lambda_{\max }(\bar{v})}{\lambda_{\min }(\bar{v})}+\frac{\sigma^{2}}{(1-\theta)\left(1+\sqrt{1-\sigma^{2}}\right)} \leqslant \tau .
$$

The left-hand side of the above inequality is monotonically increasing with respect to $\sigma$, which implies that

$$
\begin{aligned}
& \frac{\theta \sqrt{r}}{1-\theta} \frac{\lambda_{\max }(\bar{v})}{\lambda_{\min }(\bar{v})}+\frac{\sigma^{2}}{(1-\theta)\left(1+\sqrt{1-\sigma^{2}}\right)} \\
& \quad \leqslant \frac{\theta \sqrt{r}}{1-\theta} \frac{\lambda_{\max }(\bar{v})}{\lambda_{\min }(\bar{v})}+\frac{\tau^{2}}{(1-\theta)\left(1+\sqrt{1-\tau^{2}}\right)} .
\end{aligned}
$$

Thus, $\sigma\left(v^{+}, \bar{v}^{+}\right) \leqslant \tau$ is satisfied if

$$
\frac{\theta \sqrt{r}}{1-\theta} \frac{\lambda_{\max }(\bar{v})}{\lambda_{\min }(\bar{v})}+\frac{\tau^{2}}{(1-\theta)\left(1+\sqrt{1-\tau^{2}}\right)} \leqslant \tau .
$$

At this stage, if we set $\tau=\frac{1}{2}$ and $\theta=\frac{\lambda_{\min }(\bar{v})}{4 \sqrt{r} \lambda_{\max }(\bar{v})}$, the inequality (5.5) for $r \geqslant 2$ certainly holds. This means that $(x, s)$ is strictly feasible and $\sigma(v, \bar{v}) \leqslant \tau$ are maintained during the algorithm. Thus, the algorithm is well-defined.

Observe that $\frac{\lambda_{\max }(\bar{v})}{\lambda_{\min }(\bar{v})}=\frac{\lambda_{\max }\left(\bar{v}^{0}\right)}{\lambda_{\min }\left(\bar{v}^{0}\right)}$ for all iterates produced by the algorithm. Thus, an immediate result of discuss above is that for $\theta=\frac{\lambda_{\min }\left(\bar{v}^{0}\right)}{4 \sqrt{\bar{r}} \lambda_{\max }\left(\bar{v}^{0}\right)}$ the conditions $x, s \in$ int $\mathcal{K}$ and $\sigma(v, \bar{v}) \leqslant \frac{1}{2}$ are maintained throughout the algorithm. Hence, the algorithm is well-defined.

\subsection{Complexity Bound}

We proceed by considering the reduction of the duality gap in the algorithm. Recall from Lemma 5.4 that after a full NT-step the duality gap included in its target value. So we only need to consider successive target values $\|\bar{v}\|_{F}^{2}$. Using this, we prove the following theorem.

Theorem 5.2 Assume that the step size $\theta$ has its default value $\frac{\lambda_{\min }\left(\bar{v}^{0}\right)}{4 \sqrt{r} \lambda_{\max }\left(\bar{v}^{0}\right)}$. Let $\left\|\bar{v}^{0}\right\|_{F}:=\sqrt{\operatorname{Tr}\left(x^{0} \circ s^{0}\right)}$.Then, after at most

$$
\mathcal{O}\left(\sqrt{r} \frac{\lambda_{\max }\left(\bar{v}^{0}\right)}{\lambda_{\min }\left(\bar{v}^{0}\right)} \log \frac{\operatorname{Tr}\left(x^{0} \circ s^{0}\right)}{\epsilon}\right)
$$

iterations the algorithm stops, and we obtain a primal-dual interior feasible pair $(x, s)$ that satisfies $\operatorname{Tr}(x \circ s) \leqslant \epsilon$. 
Proof At the start of the algorithm the duality gap is given by

$$
\left\|\bar{v}^{0}\right\|_{F}^{2}=\operatorname{Tr}\left(x^{0} \circ s^{0}\right) \text {. }
$$

After $k$ iterations we get $\bar{v}=(1-\theta)^{k} \bar{v}^{0}$. Using Lemma 5.4 we obtain

$$
\operatorname{Tr}\left(x^{k} \circ s^{k}\right) \leqslant\|\bar{v}\|_{F}^{2}=(1-\theta)^{2 k}\left\|\bar{v}^{0}\right\|_{F}^{2}=(1-\theta)^{2 k} \operatorname{Tr}\left(x^{0} \circ s^{0}\right) .
$$

Then, the inequality $\operatorname{Tr}\left(x^{k} \circ s^{k}\right) \leqslant \epsilon$ holds if

$$
(1-\theta)^{2 k} \operatorname{Tr}\left(x^{0} \circ s^{0}\right) \leqslant \epsilon .
$$

Taking the logarithm and using $\log (1-\theta) \leqslant-\theta, 0<\theta<1$, we observe that the above inequality holds if

$$
k \geqslant \frac{1}{2 \theta} \log \frac{\operatorname{Tr}\left(x^{0} \circ s^{0}\right)}{\epsilon} .
$$

Substitution of the default value for $\theta$, we find that

$$
k \geqslant 2 \sqrt{r} \frac{\lambda \max \left(\bar{v}^{0}\right)}{\lambda_{\min }\left(\bar{v}^{0}\right)} \log \frac{\operatorname{Tr}\left(x^{0} \circ s^{0}\right)}{\epsilon} .
$$

This gives the result.

Remark 5.1 If $(\bar{v})^{2}=\mu e$, then $\frac{\lambda_{\max }\left(\bar{v}^{0}\right)}{\lambda_{\min }\left(\bar{v}^{0}\right)}=1$, and we get the iteration bound

$$
\mathcal{O}\left(\sqrt{r} \log \frac{\operatorname{Tr}\left(x^{0} \circ s^{0}\right)}{\epsilon}\right)
$$

This is matched with the iteration bound obtained in [25].

\section{Numerical Results}

In this section we solve the following LO problem using our algorithm with $\epsilon=10^{-4}$.

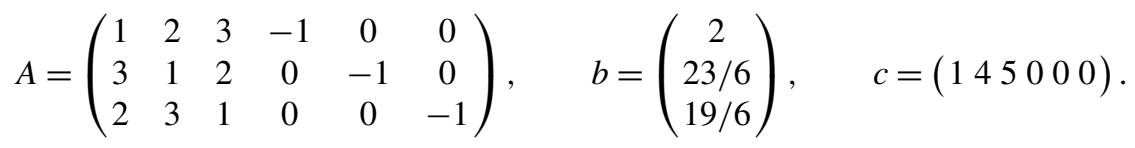

The initial primal-dual point is

$$
x^{0}=(1,1 / 2,1 / 3,1,1 / 3,2 / 3)^{\mathrm{T}}, \quad y^{0}=0, \quad s^{0}=(1,4,5)^{\mathrm{T}} .
$$

For our algorithm, we need 367 iterations to reach our accuracy. 


\section{Conclusion}

In this paper, we have developed a new weighted-path-following algorithm for solving (CP) and (CD) problems based on Darvay's technique. We have defined a new class of search directions. By employing Euclidean Jordan Algebras, we derived the complexity bound.

Some interesting topics for further research remain. The search direction used in this paper is based on the NT-scaling scheme. It may be possible to design similar algorithm using other scaling scheme and to obtain polynomial-time iteration bounds. Another topic for further research may be the development of full NT-step infeasible interior-point algorithm.

Acknowledgements The author is grateful to the two anonymous referees and the Editors for their constructive comments and suggestions to improve the presentation.

\section{References}

[1] Achache, M.: A weighted-path-following method for the linear complementarity problem. Stud. Univ. Babeş-Bolyai, Inform. XLIX(1), 61-73 (2004)

[2] Chua, C.B.: $T$-algebras and linear optimization over symmetric cones. Research report, Division of Mathematical Science, School of Physical and Mathematical Sciences, Nanyang Technological University, Singapore (2008)

[3] Darvay, Z.: New interior-point algorithms in linear programming. Adv. Model. Optim. 5(1), 51-92 (2003)

[4] Darvay, Z.: A weighted-path-following method for linear optimization. Stud. Univ. Babeş-Bolyai, Inform. XLVII(2), 3-12 (2002)

[5] Ding, J., Li, T.Y.: An algorithm based on weighted logarithmic barrier functions for linear complementarity problems. Arab. J. Sci. Eng. 15(4), 679-685 (1990)

[6] Faraut, J., Korányi, A.: Analysis on Symmetric Cones. Oxford Mathematical Monographs. Clarendon Press/Oxford University Press, New York (1994)

[7] Faybusovich, L.: A Jordan-algebraic approach to potential-reduction algorithms. Math. Z. 239(1), 117-129 (2002)

[8] Faybusovich, L.: Linear systems in Jordan algebras and primal-dual interior-point algorithms. J. Comput. Appl. Math. 86, 149-175 (1997)

[9] Faybusovich, L.: Euclidean Jordan algebras and interior-point algorithms. Positivity 1(4), 331-357 (1997)

[10] Güler, O.: Barrier functions in interior-point methods. Math. Oper. Res. 21(4), 860-885 (1996)

[11] Jansen, B., Roos, C., Terlaky, T., Vial, J.-P.: Primal-dual target-following algorithms for linear programming. Ann. Oper. Res. 62, 197-231 (1996)

[12] Kheirfam, B.: A new infeasible interior-point method based on Darvay's technique for symmetric optimization. Ann. Oper. Res. (2013). doi:10.1007/s10479-013-1474-5

[13] Kojima, M., Shindoh, S., Hara, S.: Interior-point methods for the monotone semidefinite linear complementarity problem in symmetric matrices. SIAM J. Optim. 7, 86-125 (1997)

[14] Nesterov, Y.E., Todd, M.J.: Self-scaled barriers and interior-point methods for convex programming. Math. Oper. Res. 22(1), 1-42 (1997)

[15] Nesterov, Y.E., Todd, M.J.: Primal-dual interior-point methods for self-scaled cones. SIAM J. Optim. 8(2), 324-364 (1998)

[16] Schmieta, S.H., Alizadeh, F.: Extension of primal-dual interior-point algorithm to symmetric cones. Math. Program. 96(3), 409-438 (2003)

[17] Schmieta, S.H., Alizadeh, F.: Associative and Jordan algebras and polynomial time interior-point algorithms for symmetric cones. Math. Oper. Res. 26, 543-564 (2001)

[18] Sturm, J.F.: Similarity and other spectral relations for symmetric cones. Algebra Appl. 312(1-3), 135-154 (2000) 
[19] Rangarajan, B.K.: Polynomial convergence of infeasible interior-point methods over symmetric cones. SIAM J. Optim. 16(4), 1211-1229 (2006)

[20] Roos, C., Den Hertog, D.: A polynomial method of approximate weighted centers for linear programming. Technical Report 89-13, Faculty of Technical Mathematics and Informatics, TU Delft, NL-2628 BL, Delft, The Netherlands (1994)

[21] Vieira, M.V.C.: Jordan algebraic approach to symmetric optimization. Ph.D. Thesis, Electrical Engineering, Mathematics and Computer Science, Delft University of Technology, The Netherlands (2007)

[22] Wang, G.Q.: A new polynomial interior-point algorithm for the monotone linear complementarity problem over symmetric cones with full NT-steps. Asia-Pac. J. Oper. Res. 29(2), 1250015 (2012) (pp. 20)

[23] Wang, G.Q., Bai, Y.Q.: A primal-dual path-following interior-point algorithm for second-order cone optimization with full Nesterov-Todd step. Appl. Math. Comput. 215(3), 1047-1061 (2009)

[24] Wang, G.Q., Bai, Y.Q.: A new primal-dual path-following interior-point algorithm for semidefinite optimization. J. Math. Anal. Appl. 353, 339-349 (2009)

[25] Wang, G.Q., Bai, Y.Q.: A new full Nesterov-Todd step primal-dual path-following interior-point algorithm for symmetric optimization. J. Optim. Theory Appl. 154(3), 966-985 (2012)

[26] Wang, G.Q., Yue, Y.J., Cai, X.Z.: A weighted-path-following method for monotone horizontal linear complementarity problem. Fuzzy Inf. Eng. 54, 479-487 (2009)

[27] Wang, G.Q., Yu, C.J., Teo, K.L.: A full Nesterov-Todd step feasible interior-point method for convex quadratic optimization over symmetric cone. Appl. Math. Comput. 221(15), 329-343 (2013) 